\title{
How can gastro-intestinal tuberculosis diagnosis be improved? A prospective cohort study
}

Christopher Lowbridge ${ }^{1 *}$, Soraya A. M. Fadhil², Gayathri D. Krishnan², Emma Schimann³, Raman Muthu Karuppan², Nagaraj Sriram², Giri Shan Rajahram², Jayaram Menon ${ }^{2}$, Aatish Patel ${ }^{3}$, Timothy William, ${ }^{3,4}$, Dawn Carmel Paul ${ }^{5}$ and Anna P. Ralph ${ }^{1,3}$

\begin{abstract}
Background: Gastrointestinal tuberculosis (TB) is diagnostically challenging; therefore, many cases are treated presumptively. We aimed to describe features and outcomes of gastrointestinal TB, determine whether a clinical algorithm could distinguish TB from non-TB diagnoses, and calculate accuracy of diagnostic tests.

Methods: We conducted a prospective cohort study of hospitalized patients in Kota Kinabalu, Malaysia, with suspected gastrointestinal TB. We recorded clinical and laboratory characteristics and outcomes. Tissue samples were submitted for histology, microscopy, culture and GeneXpert MTB/RIF ${ }^{\oplus}$. Patients were followed for up to 2 years.
\end{abstract}

Results: Among 88 patients with suspected gastrointestinal TB, 69 were included in analyses; 52 (75\%) had a final diagnosis of gastrointestinal TB; 17 had a non-TB diagnosis. People with TB were younger (42.7 versus 61.5 years, $p=0.01$ ) and more likely to have weight loss (91\% versus $64 \%, p=0.03$ ). An algorithm using age $<44$, weight loss, cough, fever, no vomiting, albumin $>26 \mathrm{~g} / \mathrm{L}$, platelets $>340 \times 10^{9} / \mathrm{L}$ and immunocompromise had good specificity (96.2\%) in predicting TB, but very poor sensitivity (16.0\%). GeneXpert ${ }^{\oplus}$ performed very well on gastrointestinal biopsies (sensitivity $95.7 \%$ versus $35.0 \%$ for culture against a gold standard composite case definition of confirmed TB). Most patients (79\%) successfully completed treatment and no treatment failure occurred, however adverse events (21\%) and mortality (13\%) among TB cases were high. We found no evidence that 6 months of treatment was inferior to longer courses.

Conclusions: The prospective design provides important insights for clinicians managing gastrointestinal TB. We recommend wider implementation of high-performing diagnostic tests such as GeneXpert ${ }^{\oplus}$ on extra-pulmonary samples.

Keywords: Gastro-intestinal tuberculosis, Extra-pulmonary tuberculosis, Cohort study, Diagnosis, GeneXpert

\footnotetext{
* Correspondence: christopher.lowbridge@menzies.edu.au

'Global and Tropical Health Division, Menzies School of Health Research,

Charles Darwin University, Box 41096, Casuarina NT, Darwin, PO 0811,

Australia

Full list of author information is available at the end of the article
}

(C) The Author(s). 2020 Open Access This article is licensed under a Creative Commons Attribution 4.0 International License, which permits use, sharing, adaptation, distribution and reproduction in any medium or format, as long as you give appropriate credit to the original author(s) and the source, provide a link to the Creative Commons licence, and indicate if changes were made. The images or other third party material in this article are included in the article's Creative Commons licence, unless indicated otherwise in a credit line to the material. If material is not included in the article's Creative Commons licence and your intended use is not permitted by statutory regulation or exceeds the permitted use, you will need to obtain permission directly from the copyright holder. To view a copy of this licence, visit http://creativecommons.org/licenses/by/4.0/ The Creative Commons Public Domain Dedication waiver (http://creativecommons.org/publicdomain/zero/1.0/) applies to the data made available in this article, unless otherwise stated in a credit line to the data. 


\section{Background}

Gastrointestinal tuberculosis (TB) is a relatively uncommon form of $\mathrm{TB}$ which is defined as infection of the peritoneum, abdominal organs or abdominal lymphatic system [1]. TB control programs typically focus on pulmonary $\mathrm{TB}$ which is the predominant form of the disease; extra-pulmonary $\mathrm{TB}$, in particular gastro-intestinal $\mathrm{TB}$, is relatively neglected. This is likely due to it being more difficult to diagnose and lacking the transmission potential of smear-positive pulmonary TB. However, extra-pulmonary TB, including gastrointestinal $\mathrm{TB}$ often represents the worst end of the TB disease severity spectrum, with poorer prognosis and treatment outcomes [2]. Severe and potentially life-threatening complications of gastrointestinal TB include intestinal strictures, obstruction, perforation and bleeding [3].

$\mathrm{TB}$ is a disease of major public health importance in Malaysia. Sabah State, located in Malaysian Borneo, has a particularly high burden of disease, accounting for approximately 20 to $30 \%$ of Malaysia's TB cases despite having only $10 \%$ of Malaysia's population $[4,5]$.

Gastrointestinal TB is particularly difficult to diagnose, with only a minority of cases able to be microbiologically confirmed. This creates risks of both under and overdiagnosis, uncertainty about best clinical approaches, and potential under-reporting of cases to the national TB register. These diagnostic difficulties in Sabah are compounded by the presence of important differential diagnoses which can mimic the various manifestations of gastrointestinal TB, including infectious causes (e.g. Burkholderia pseudomallei), [6] and non-infectious causes (inflammatory bowel disease, lymphoma other malignancies).

This study describes the presentation, diagnosis, management and short and long-term outcomes of gastrointestinal TB cases at the Queen Elizabeth Hospital and Women's and Children's Hospital in Sabah, Malaysia. Through analysis of presenting signs and symptoms, clinical evaluation and diagnostic results, we aimed to develop a clinical algorithm to aid in the diagnosis of gastrointestinal TB. A clinical algorithm or score could help categorize patients as having gastrointestinal TB or an alternative diagnosis while awaiting, or in the absence of, further diagnostic information. Further we estimated the sensitivity and specificity of diagnostic tests, including GeneXpert ${ }^{\ominus}$ for laboratory confirmation of gastrointestinal TB.

\section{Methods}

\section{Study setting and design}

We conducted a prospective, observational study of the presentation, diagnosis, management and outcomes of gastrointestinal TB cases presenting to Queen Elizabeth
Adult Hospital, Kota Kinabalu, Sabah, Malaysia. Sequential patients presenting to Queen Elizabeth Hospital with suspected gastrointestinal TB and referred to the Departments of Medicine, Surgery, Gastroenterology or Infectious Diseases were eligible for inclusion. Diagnostic work-up of patients presenting with suspected gastrointestinal TB included blood, microbiological, histopathological and other tests (see supplementary material Table 1). Patients were classified as 'confirmed', 'probable', or 'possible gastrointestinal TB', or as 'not TB' using standard case definitions (see supplementary material Table 2).

Samples were processed at the research laboratory at Queen Elizabeth Hospital (GeneXpert MTB/RIF ${ }^{\oplus}$ ), the pathology department of Queen Elizabeth Hospital (histology) and the Public Health Laboratory of Kota Kinabalu, Malaysia (culture, TB polymerase chain reaction [PCR]). Mycobacterial culture was performed using Ogawa media. PCR was performed using the Seegene ${ }^{\text {TM }}$ Anyplex MTB/NTM kit (estimated sensitivity versus culture: 100\%) [7]. To prepare tissue samples for GeneXpert testing, samples were handled in a biosafety cabinet and were homogenized using a sterile mortar and pestle or chopped / mashed finely in a sterile petri dish with a scalpel blade. Approximately $1 \mathrm{~mL}$ sterile saline was added. A sterile disposable pipette was used to transfer the material to a tube for mixing and incubation with the GeneXpert buffer ( $1 \mathrm{~mL}$ sample to $2 \mathrm{~mL}$ buffer). After 15 min of mixing and incubation, a sterile disposable pipette was used to transfer only liquid (no particulate matter) into the MTB/RIF cartridge.

Participants with definite, probable or possible gastrointestinal $\mathrm{TB}$ that was proven or assumed to be fully drug susceptible were treated using a standard regimen consisting of isoniazid, rifampicin, ethambutol, and pyrazinamide for a duration of at least 6 months, in accordance with World Health Organization (WHO) and Malaysian Department of Health guidelines [8]. Divergence from the standard regimen was made at the discretion of the treating physician, due to factors such as deranged liver function tests at baseline or intolerance to one or more of the standard first-line medications.

Participants with confirmed or suspected drugresistant $\mathrm{TB}$ received a regimen and treatment duration that was consistent with WHO guidelines for drugresistant $\mathrm{TB}$, which includes the recommendation for continuation of treatment for 18-months after culture conversion [9].

Follow-up of patients with gastrointestinal TB was conducted in line with standard clinical practice at the study sites. This consisted of monthly follow-up for the first 6 months, followed by follow-up every three to four months up to two years post treatment, to monitor for relapse of $\mathrm{TB}$. 
A standardized data sheet was used to collect demographic and epidemiological information, clinical history, vital, biochemical and microbiological data, treatment, outcome, and final diagnosis. Data were entered into a REDCap electronic data capture tool [10] hosted at Menzies School of Health Research and analyzed using STATA $^{\circ}$ version 15 . Two levels of analysis were conducted, the first comparing confirmed and probable gastrointestinal TB against patients classified as non-TB, the second level limited to comparing confirmed TB against non-TB. Outcomes of a six-month treatment regimen were assessed by determining adverse events, requirement to change treatment regimen, short-term (end of treatment) outcome and two-year relapse rates.

\section{Statistical analyses}

Pearson's $\chi^{2}$ test was used to assess the difference in distribution of categorical variables between groups. Welch's unequal variances t-test or Kruskal-Wallis equality of populations rank test were used for analysis of difference in means of continuous variables between groups. Sensitivity and specificity of Mycobacterium tuberculosis culture, polymerase chain reaction (PCR) and GeneXpert $\mathrm{MTB} / \mathrm{RIF}^{\circ}$ as well as histology for detecting presence of $M$. tuberculosis were calculated using the confirmed TB case definition as gold standard. Histology results were blindly reviewed by three infectious diseases physicians and classified as 'highly suggestive of TB', 'suggestive of TB', 'not suggestive of TB' or 'clear alternative diagnosis'. Disagreements were resolved by discussion until consensus was reached.

To develop a clinical algorithm to support diagnosis of gastrointestinal TB, we calculated the odds ratios and log odds of relevant variables (age, symptomatology, duration of symptoms, liver function tests, blood chemistry and blood count), comparing probable and confirmed TB cases to confirmed non-TB cases. We excluded variables where the $P$-value of the odds ratio was $\geq 0.5$ and limited variables for inclusion in the model to the top eight variables based on highest absolute log odds value. Points were allocated to each of the variables in the algorithm based on log odds value, and area under the curve of receiver operator characteristics was calculated to assess sensitivity, specificity and positive predictive value of the algorithm, using final diagnosis of $\mathrm{TB}$ as the gold standard.

\section{Results}

\section{Patient characteristics}

From a total of 88 patients suspected of having gastrointestinal TB on presentation at Queen Elizabeth Hospital and the Women's and Children's Hospitals, Kota Kinabalu, Sabah, between March 2015 and June 2017, consent for inclusion in the study was obtained from 77 patients. Of these, eight patients were excluded from the analysis due to incomplete follow-up and final diagnosis. Among the 69 patients included in the study: 52 (75\%) had a final diagnosis of gastrointestinal TB (25 confirmed, 13 probable, 14 possible cases), and 17 patients were assessed to have non-TB diagnoses, the majority of which $(n=12)$ were malignancy (Supplementary Fig. 1$)$.

Among the 52 cases of confirmed, probable and possible gastrointestinal TB, 42 (81\%) had infection of the intestine, 14 (27\%) had infection of an abdominal lymph node, 13 (25\%) had infection of the peritoneum, 8 (15\%) had infection of the liver, and $1(2 \%)$ had biliary infection (Fig. 1). A total of 45 (87\%) gastrointestinal TB cases were recorded as having involvement of multiple sites or organs within the gastrointestinal system.

The mean age of patients with a final diagnosis of gastrointestinal TB (confirmed, probable, possible) was 42.7 years, significantly lower when compared with the mean age of 61.5 years for patients with non-TB diagnosis $(t$ (46 d.f.) $=5.07, p<0.01)$ (Fig. 2). There was no significant difference in sex, with $51 \%$ of gastrointestinal TB cases being male, compared with $60 \%$ of non-TB cases.

\section{Microbiological and histological results}

A total of 110 biopsy samples were collected from patients during initial work-up and ongoing clinical followup. The largest proportion of samples were collected from the large bowel (34\%), followed by the ileum (14\%) and peritoneum (10\%). Samples were also collected from the stomach, small bowel, liver and omentum. Peritoneal biopsy samples were collected predominantly via laparoscopy $(n=7)$ or laparotomy $(n=2)$.

Twenty-four of $42 \mathrm{~TB}$ cases tested by GeneXpert ${ }^{\circ}$ were positive for M.tuberculosis. Of $39 \mathrm{~TB}$ cases with mycobacterial culture results, 12 had M.tuberculosis detected by culture. GeneXpert ${ }^{\circ}$ positivity by sample type is detailed in supplementary Table 4. Rifampicin resistance was detected by GeneXpert ${ }^{\circ}$ in three cases, of which one was found to be sensitive to rifampicin and another was confirmed rifampicin resistant by phenotypic drugsusceptibility testing. The third was treated as rifampicin resistant based on the GeneXpert ${ }^{\circ}$ result. Two cases were found to be isoniazid mono-resistant by phenotypic drug-susceptibility testing.

Sensitivity and specificity of diagnostic tests for M.tuberculosis are presented in Table 1. GeneXpert ${ }^{\circ}$, culture, PCR and smear microscopy for Acid Fast Bacilli all achieved $100 \%$ specificity against the gold standard of final case classification of confirmed TB. GeneXpert ${ }^{\circ}$ achieved high sensitivity (95.7\%), compared with very low sensitivity of culture (35.0\%), PCR (50.0\%) and microscopy (31.0\%). Histology achieved moderate sensitivity $(68.0 \%)$ and specificity (77.1\%) when using the lower 


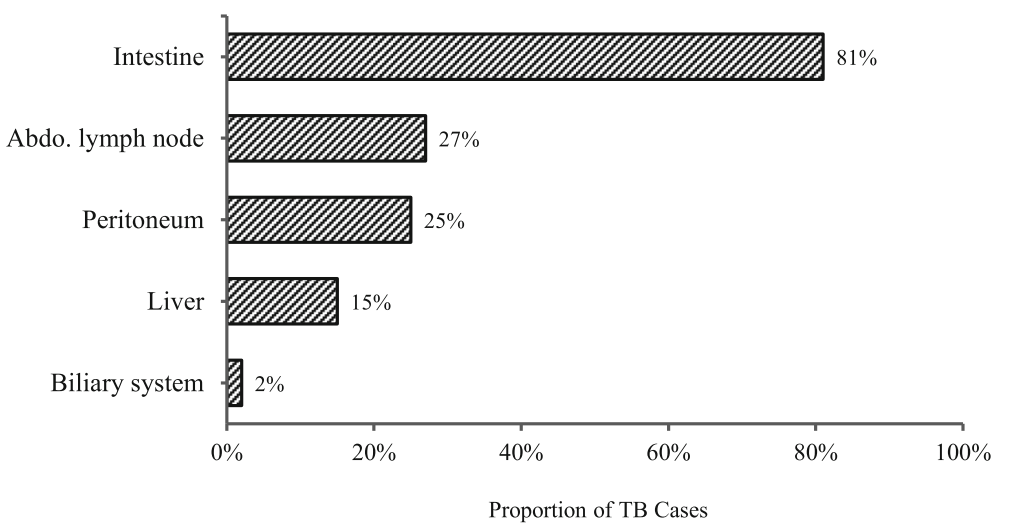

Fig. 1 Site of gastrointestinal TB disease

threshold for evidence of TB ('suggestive' or 'highly suggestive' of TB).

\section{Features distinguishing TB from non-TB and development} of a clinical score

Among confirmed and probable TB cases, the most common symptoms reported at presentation were weight loss (91\%), fever (54\%) and abdominal pain (52\%). For non-TB cases, the most common presenting symptom was abdominal pain (71\%), followed by weight loss (64\%) and vomiting (36\%) (Fig. 3). Confirmed and probable TB cases were significantly more likely to present with weight loss compared with non-TB cases $\left(\chi^{2}=4.93, p=0.03\right)$. The proportion of probable and confirmed TB cases with a recorded temperature of 38 degrees Celsius or higher on presentation was higher $(24 \%)$ than non-TB cases $(14 \%)$, however the difference was not statistically significant. Confirmed and probable TB cases presented with longer median duration of fever (4 weeks vs. 1 week) and abdominal pain (8 vs. 4 weeks) but shorter duration of weight loss (10 vs. 14 weeks), vomiting (3 vs. 18 weeks), cough (8 vs. 53 weeks) and fatigue (8 vs. 12 weeks), compared with non-TB cases. However, none of the differences in duration of symptoms between $\mathrm{TB}$ and non-TB cases were statistically significant.

Two patients in our study cohort had HIV infection, both with confirmed gastrointestinal TB. There were no statistically significant differences in the proportion of patients who had: a history of Bacillus Calmette-Guérin (BCG) vaccination; past TB diagnosis; family history of TB; positive tuberculin skin test; HIV infection; diabetes; or immunocompromising condition, between confirmed $\mathrm{TB}$ cases and non-TB cases or confirmed and probable TB cases and non-TB cases. No significant risk factors emerged when limiting the analysis to either confirmed cases of TB or non-TB cases with malignancy.

There were no statistically significant differences in blood chemistry, blood count or liver function tests

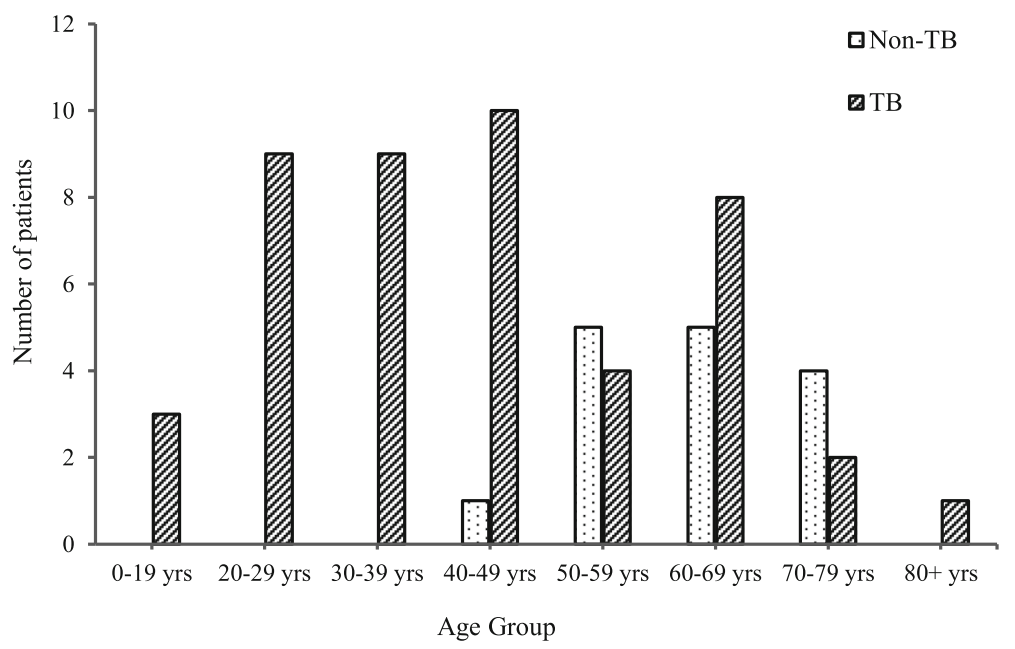

Fig. 2 Age distribution of patients by final diagnosis 
Table 1 Sensitivity and specificity of diagnostic tests using gastrointestinal biopsy specimens

\begin{tabular}{llllll}
\hline & $\boldsymbol{N}$ tests & Sensitivity \% & Specificity \% & PPV \% & NPV \% \\
\hline GeneXpert $^{\oplus}$ & 57 & 95.7 & 100 & 100 & 97.1 \\
Culture & 42 & 35.0 & 100 & 100 & 62.9 \\
PCR & 29 & 50.0 & 100 & 100 & 68.2 \\
AFB & 55 & 31.0 & 100 & 100 & 56.5 \\
Histology $^{\text {a }}$ & 60 & 68.0 & 77.1 & 68.0 & 77.1 \\
Histology $^{\text {b }}$ & 60 & 36.0 & 85.7 & 64.3 & 65.2
\end{tabular}

${ }^{a}$ Histology results classified as 'suggestive' or 'highly suggestive of TB' versus results classified as not 'suggestive of $\mathrm{TB}^{\prime}$ or 'clear alternative diagnosis'

${ }^{b}$ Histology results classified as 'highly suggestive of TB' versus results classified as 'clear alternative diagnosis'

between confirmed and probable $\mathrm{TB}$ cases and non-TB cases (supplementary material, Table 3 ). Non-TB cases had lower mean albumin levels $(21.54$ vs. $26.17 \mathrm{~g} / \mathrm{L})$ and higher mean bilirubin levels ( 24.0 vs. $13.35 \mathrm{mmol} / \mathrm{L})$ from peripheral blood samples on initial diagnostic work-up-however these differences were not significant. No statistically significant differences emerged when limiting the analysis to confirmed TB cases or non-TB malignancy. Blood cultures were positive in four of four non-TB cases who had blood cultures collected (isolates were: Elizabethkingia meningoseptica, Escherichia coli, and two with Klebsiella pneumoniae) and one probable TB case (Acinetobacter baumannii).

A composite clinical score was developed to determine whether a scoring system could reliably distinguish TB from non-TB diagnosis (Table 2). The best performing algorithm for diagnosis of gastrointestinal TB included: age less than 44 years; weight loss; cough; fever; no vomiting; blood albumin level great than $26 \mathrm{~g} / \mathrm{L}$; platelet count greater than $340 \times 10^{9} / \mathrm{L}$; and history of immunocompromising illness/treatment. However, this algorithm achieved an area under the receiver operator characteristics curve of just 0.5873. The highest correct classification of cases $(70.1 \%)$ occurred at a cut-point of eight points from a maximum of 11 points, this corresponded to a specificity of $96.2 \%$, but sensitivity of only $16.0 \%$ (positive predictive value $=60.0 \%$; negative predictive value $=3.8 \%$ ). There was no significant difference in performance of the composite clinical score when assessed against an alternative gold standard, such as microbiologically confirmed M.tuberculosis.

\section{Treatment}

Details of the prescribed anti-tuberculous treatment regimen was documented for 38 patients. Standard firstline treatment of isoniazid, rifampicin, ethambutol and pyrazinamide was initiated in $32(84 \%)$ TB cases with documentation of treatment, with proposed treatment durations at initiation of, six-months, nine-months and 12-months for 9 (28\%), $16(50 \%)$ and $7(22 \%)$ cases respectively. Most patients (91\%) received pyridoxine (vitamin B6) during treatment. Six patients were given an alternative regimen: one due to adverse reaction to ethambutol, one due to baseline liver impairment, two due to detection of rifampicin resistance and two for an undocumented reason. Of the two cases with rifampicin resistance: one was treated with a regimen consisting of kanamycin, ethionamide, cycloserine and levofloxacin; the other with a regimen consisting of isoniazid, ethambutol, pyrazinamide, kanamycin, levofloxacin and ethionamide. Mean treatment duration at completion for cases receiving first-line regimens was 8.8 months (median 9.0 months, maximum 12.4 months).

A total of 12 adverse events were reported among eight patients undergoing anti-tuberculous treatment. The majority (67\%) of adverse events occurred during the first 2 months of treatment. Hepatotoxicity and rash

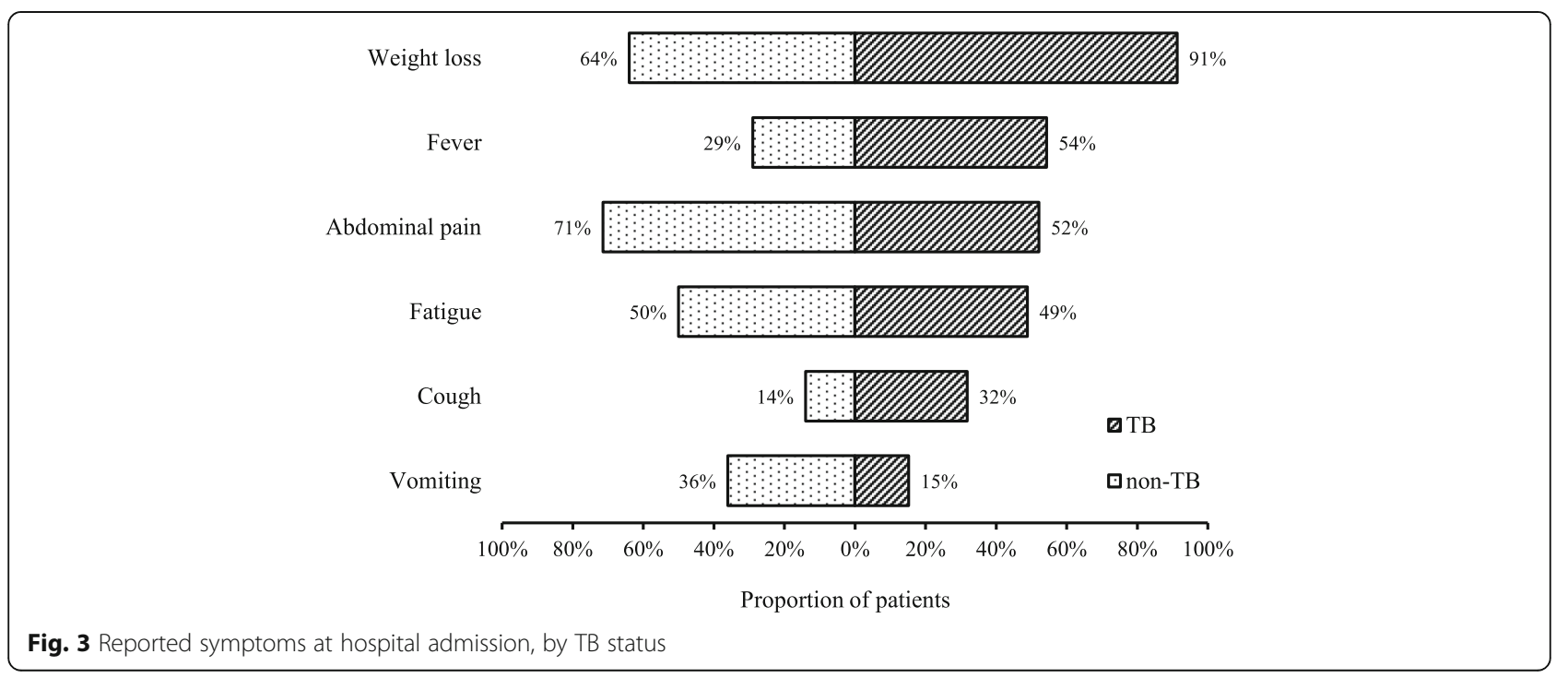


Table 2 Clinical score tested for diagnosis of gastrointestinal tuberculosis

\begin{tabular}{ll}
\hline Criteria & Points \\
\hline Age less than 44 years & 3 \\
Weight loss & 2 \\
No vomiting & 1 \\
Albumin $>26 \mathrm{~g} / \mathrm{L}$ & 1 \\
Platelets $>340 \times 10^{9} / \mathrm{L}$ & 1 \\
Cough & 1 \\
Immunocompromised & 1 \\
Fever $>38^{\circ} \mathrm{C}$ & 1 \\
Total possible score & 11 \\
\hline
\end{tabular}

were the most frequent adverse events reported, accounting for $25 \%$ of adverse events each. Most adverse events were mild or moderate (75\%), three required hospitalization but none were life-threatening or resulting in death. Changes in treatment regimen were required in six (16\%) of the 38 patients for whom treatment details were available. Five adverse events resulted in permanent cessation of a drug, two resulted in a drug being stopped and then reintroduced and one resulted in a change in route of administration of a drug. No new agents were added to any regimens because of an adverse event.

TB cases were followed-up for a median of 7.1 months (maximum 16.9 months). Final outcome was recorded for $92 \%$ of gastrointestinal TB cases. Of these, $79 \%$ successfully completed treatment or were cured; no case of treatment failure was recorded. A total of six (13\%) TB cases died. TB cases who died had a mean age of 54 years and all deaths occurred in the first 5 months following hospitalization. Four cases $(8 \%)$ defaulted from treatment. Three (18\%) non-TB cases died (two with malignancy and one with Helicobacter pylori gastritis).

\section{Discussion}

In this two-year prospective study, we found that $75 \%$ of people presenting with suspected gastrointestinal TB had a final diagnosis of TB. A composite clinical score using patient characteristics and investigation findings had good specificity in predicting a diagnosis of TB, but poor sensitivity. This highlights the importance of access to reliable diagnostic tests for diagnosing extrapulmonary TB. We identified that GeneXpert MTB/RIF performed very well on tissue samples and was considerably superior to culture in this specific setting. This reflects a pragmatic real-world situation rather than a laboratory based head-to-head comparison of diagnostic modalities, taking into account challenges in reporting results back to treating clinicians. Other key findings from this cohort were the relatively high rate of adverse treatment events necessitating regimen changes in 16\% of individuals, and the high mortality rate among $\mathrm{TB}$ cases of $13 \%$. The unique study design using prospective enrolment of suspected TB cases provides important new insights for clinicians managing this condition especially with regards to diagnostic and prognostic features. The findings highlight the ongoing morbidity and mortality burden posed by TB in this endemic area.

It is well established that gastrointestinal TB is diagnostically challenging due to cases commonly presenting with non-specific symptoms, laboratory, and radiological findings $[11,12]$. This is especially the case in a setting such as Sabah where numerous infections which may present similarly to TB are endemic, in addition to common non-infectious differential diagnoses of gastrointestinal $\mathrm{TB}$, such as malignancy and autoimmune conditions. Delayed diagnosis can result in increased risk of complications or worse patient outcomes [13]. Findings from our prospective observational study highlight the challenge of diagnosing gastrointestinal TB. We found substantial similarities in demographic and risk factor profiles, clinical presentations and basic laboratory findings during initial assessment and work-up.

A robust algorithm for classifying gastrointestinal $\mathrm{TB}$ cases, as is available for TB meningitis, [14] based on scoring of clinical information would potentially improve the early diagnosis of gastrointestinal TB cases. We attempted to develop such an algorithm, based on the constellation of signs and symptoms listed in Table 2 . While a high score using this algorithm was suggestive of gastrointestinal TB, its poor sensitivity and predictive values make it inadequate for use as a clinical or research classification tool.

Given the inherent challenges in clinical diagnosis and classification of gastrointestinal $\mathrm{TB}$, the use of rapid diagnostics is important. The GeneXpert ${ }^{\circ} \mathrm{MTB} /$ RIF assay has proved a hugely useful test in the diagnosis of pulmonary $\mathrm{TB}$. In the diagnosis of extrapulmonary $\mathrm{TB}$, it has been shown to be specific, however concerns have been raised about its sub-optimal sensitivity [15]. We found that GeneXpert ${ }^{\circ}$ performed well on biopsy samples, being both highly sensitive and specific. This is in keeping with a study by Hilleman et al., which found high sensitivity and specificity of GeneXpert ${ }^{\circ}$ MTB assay from gastric fluid and stool specimens $(87.5 \%$ \& 100 , and $100 \%$ and $98.6 \%$ respectively) in cases of extra-pulmonary TB, [16] and a metanalysis of GeneXpert ${ }^{\circ}$ on extra-pulmonary samples which found a pooled sensitivity and specificity of 86 and $98 \%$ respectively from gastrointestinal samples [15]. In contrast to these findings however, two studies from India, assessing the performance of GeneXpert $^{\circ}$ on intestinal biopsies found low sensitivity of 8.1 and $32 \%$ respectively $[17,18]$. 
Caution should be applied in comparing our findings of sensitivity and specificity with other estimates, given our use of final clinical diagnosis as opposed to $M$. tuberculosis culture as the gold standard, as well as the relatively small sample sizes of published studies reporting sensitivity and specificity of GeneXpert ${ }^{\circ}$ from gastrointestinal samples. The superiority of GeneXpert ${ }^{\circ}$ over culture observed here may not be generalizable to settings with advanced expertise in $M$. tuberculosis culture (including recovery from contaminated samples) which may result in greater concordance between GeneXpert ${ }^{\circ}$ and culture results. Ogawa culture medium used at the reference laboratory may have slightly lower sensitivity for $M$. tuberculosis detection than liquid culture media or other egg-based solid media such as LowensteinJensen $[19,20]$. Also, the laboratory lacked an electronic reporting system at the time of the study - since rectified - resulting in missing culture results in four instances where a GeneXpert ${ }^{\circ}$ was available and positive.

Our findings emphasize the need for further investment in research and development of new tools to improve the diagnosis of gastrointestinal $\mathrm{TB}$, especially the identification of novel systemic biomarkers for TB infection and disease leading to the availability of new in vitro assays.

The chief limitation of the study is the sample size. While the original cohort was relatively large for a study of gastrointestinal TB, not all people within the cohort were able to be included in the analysis, and as the majority of the cohort $(75 \%)$ had a final diagnosis of $\mathrm{TB}$, the number of non-TB cases was small. The sample size calculation for a descriptive study of a dichotomous variable (the dichotomous variable being diagnosis of TB or not among presenting patients) would indicate that 288 patients would be needed to be able to make estimates with $95 \%$ confidence $(\alpha=0.05)$ if the proportion found to have TB was $75 \%$ [21]. The superiority of GeneXpert MTB/RIF over culture may not be reproducible in other international settings where culture facilities may be better supported. However, the reality is that high TBburden settings often lack high-quality, accredited TB culture facilities; therefore, the findings are relevant to such settings.

We did not identify any cases of treatment failure within our study cohort. While $13 \%$ of TB cases in our cohort died, all deaths occurred less than 6 months after starting treatment. While our study was not powered to compare treatment regimens, we found no evidence that 6 months of treatment was less effective than a longer treatment course. This is consistent with a review by the Cochrane group which, although based on small numbers of patients, found no evidence that 6 months treatment duration was inadequate for treatment of gastrointestinal TB [22].

\section{Conclusions}

Gastrointestinal TB remains diagnostically challenging. An algorithm developed to classify patients presenting with suspected gastrointestinal TB cases was specific but insensitive. Testing of such algorithms in larger populations of patients with suspected gastrointestinal TB will reveal whether a composite clinical score could help categorize patients while awaiting, or in the absence of, further diagnostic information. Wider implementation of existing high-performing diagnostic tests such as GeneXpert ${ }^{\circ}$ on extra-pulmonary samples, and ongoing investment in new diagnostics development, is needed. We recommend increased access to and uptake of GeneXpert $^{\circ}$ on gastrointestinal samples in suspected TB cases.

\section{Supplementary information}

Supplementary information accompanies this paper at https://doi.org/10. 1186/s12879-020-04983-y.

Additional file 1: Table S1. Diagnostic work-up of patients with suspected gastrointestinal tuberculosis. Table S2. Gastrointestinal tuberculosis case definitions. Figure S1. Recruitment and classification of study participants. Table S3. Mean blood test results during initial diagnostic work-up. Table S4. Gastrointestinal biopsy and ascitic fluid GeneXpert ${ }^{\oplus}$ MTB results by type and site.

\section{Abbreviations}

BCG: Bacillus Calmette-Guérin vaccine; HREC: Human Research Ethics Committee; MTB: Mycobacterium tuberculosis; NMRR: National Medical Research Register (Malaysia); PCR: Polymerase chain reaction; RIF: Rifampicin; TB: Tuberculosis; WHO: World Health Organization

\section{Acknowledgements}

We greatly thank the Director General of the Ministry of Health, Malaysia, for supporting this study. We thank Kelly Nestor, Florena Maidik and Sharon Nestor for undertaking the GeneXpert testing; Joseph Benedict and Kim Piera for logistic and laboratory support; and Dr. Ella Meumann for helping to adjudicate histopathology results.

\section{Author contributions}

JM, TW and APR conceived the study. APR and TW developed the protocol. SAMF, GDK, ES and GSR enrolled participants. SAMF, GDK, RMK, NS, ES and AP collected samples and data. DCP processed samples. CL analyzed data. $\mathrm{CL}$ and APR drafted the paper. All authors have reviewed and approved the manuscript.

\section{Funding}

Queen Elizabeth Hospital supported this study through provision of additional laboratory and radiological testing of study participants. Investigator time to conduct this study was supported by the National Health and Medical Research Council (NHMRC) of Australia (grants 605806 and 1131932). APR is supported by NHMRC fellowship 1142011. The funders had no role in study design, data collection and analysis, decision to publish, or preparation of the manuscript.

\section{Availability of data and materials}

The dataset generated and analyzed during the current study is not publicly available due to it containing identifying medical information. Condensed anonymized data are available from the corresponding author on reasonable request.

Ethics approval and consent to participate

The study was conducted in accordance with approval by the Malaysia National Medical Research Ethics Committee (NMRR-13-963-17830) and the 
Human Research Ethics Committee of the Northern Territory Top End Health Services and Menzies School of Health research (HREC-2014-2254). All study participants provided written consent to participate.

\section{Consent for publication}

Not applicable. No individual data or image is presented. All results are presented as aggregate.

\section{Competing interests}

The authors declare that they have no competing interests.

\section{Author details}

${ }^{1}$ Global and Tropical Health Division, Menzies School of Health Research, Charles Darwin University, Box 41096, Casuarina NT, Darwin, PO 0811, Australia. ${ }^{2}$ Department of Medicine, Queen Elizabeth Hospital, Kota Kinabalu, Malaysia. Infectious Diseases Society Sabah-Menzies School of Health Research Clinical Research Unit, Kota Kinabalu, Sabah, Malaysia. ${ }^{4}$ Jesselton Medical Centre, Kota Kinabalu, Malaysia. ${ }^{5}$ Public Health Laboratory (Makmal Kesihatan Awam), Kota Kinabalu, Malaysia.

Received: 5 November 2019 Accepted: 19 March 2020

Published online: 30 March 2020

\section{References}

1. Sheer TA, Coyle WJ. Gastrointestinal tuberculosis. Curr Gastroenterol Rep. 2003;5(4):273-8.

2. Ohene S-A, Bakker MI, Ojo J, Toonstra A, Awudi D, Klatser P. Extrapulmonary tuberculosis: a retrospective study of patients in Accra, Ghana. PLoS One. 2019;14(1):e0209650-e.

3. Ha HK, Ko GY, Yu ES, Yoon KH, Hong WS, Kim HR, et al. Intestinal tuberculosis with abdominal complications: radiologic and pathologic features. Abdom Imaging. 1999;24(1):32-8.

4. Chang KC, Leung CC, Grosset J, Yew WW. Treatment of tuberculosis and optimal dosing schedules. Thorax. 2011;66(11):997-1007.

5. Paul DC, Chew A, Yau NL, Hollip KC, Tangkanggau F, Lasimin S, et al. Multidrug resistant Mycobacterium tuberculosis complex: a laboratory perspective (conference poster abstract). 7th Public Health Colloquium, Sabah State Health Department; Ming Garden Hotel, Kota Kinabalu, Malaysia, 21-22 November2012. Accessed 23 Jul 2019.

6. Nathan S, Chieng S, Kingsley PV, Mohan A, Podin Y, Ooi MH, et al. Melioidosis in Malaysia: incidence, clinical challenges, and advances in understanding pathogenesis. Trop Med Infect Dis. 2018;3(1). https://www. mdpi.com/2414-6366/3/1/25.

7. Perry MD, White PL, Ruddy M. Potential for use of the Seegene Anyplex MTB/NTM real-time detection assay in a regional reference laboratory. J Clin Microbiol. 2014:52(5):1708-10.

8. World Health Organization. Treatment of tuberculosis guidelines. Geneva: World Health Organization; 2010

9. World Health Organisation. Guidelines for the programmatic management of drug-resistant tuberculosis: emergency update. Geneva: WHO; 2008. WHO/HTM/TB/2008.402, 2008: [Available from: http://whqlibdoc.who.int/ publications/2008/9789241547581_eng.pdf.

10. Harris PA, Taylor R, Thielke R, Payne J, Gonzalez N, Conde JG. Research electronic data capture (REDCap)--a metadata-driven methodology and workflow process for providing translational research informatics support. J Biomed Inform. 2009;42(2):377-81.

11. Lin YS, Huang YC, Lin TY. Abdominal tuberculosis in children: a diagnostic challenge. Journal of microbiology, immunology, and infection $=$. Wei mian yu gan ran za zhi. 2010;43(3):188-93.

12. Kentley J, Ooi JL, Potter J, Tiberi S, O'Shaughnessy T, Langmead L, et al. Intestinal tuberculosis: a diagnostic challenge. Tropical Med Int Health. 2017; 22(8):994-9.

13. Debi U, Ravisankar V, Prasad KK, Sinha SK, Sharma AK. Abdominal tuberculosis of the gastrointestinal tract: revisited. World J Gastroenterol. 2014;20(40):14831-40.

14. Marais S, Thwaites G Fau, Schoeman JF, Schoeman Jf Fau - Torok ME, Torok Me Fau - Misra UK, Misra Uk Fau - Prasad K, Prasad K Fau - Donald PR, et al. Tuberculous meningitis: a uniform case definition for use in clinical research. (1474-4457 (Electronic)). Accessed 14 Jan 2019.
15. Penz E, Boffa J, Roberts DJ, Fisher D, Cooper R, Ronksley PE, et al. Diagnostic accuracy of the Xpert(R) MTB/RIF assay for extra-pulmonary tuberculosis: a meta-analysis. Int J Tuberculosis Lung Dis. 2015;19(3):278-84 i-iii.

16. Hillemann D, Rüsch-Gerdes S, Boehme C, Richter E. Rapid molecular detection of extrapulmonary tuberculosis by the automated GeneXpert MTB/RIF system. J Clin Microbiol. 2011;49(4):1202-5.

17. Kumar S, Bopanna S, Kedia S, Mouli P, Dhingra R, Padhan R, et al. Evaluation of Xpert MTB/RIF assay performance in the diagnosis of abdominal tuberculosis. Intest Res. 2017;15(2):187-94.

18. Bellam BL, Mandavdhare HS, Sharma K, Shukla S, Soni H, Kumar-M P, et al. Utility of tissue Xpert-Mtb/Rif for the diagnosis of intestinal tuberculosis in patients with ileocolonic ulcers. Therapeutic Adv Infect Dis. 2019;6: 2049936119863939

19. Ceyhan I, Simsek H, Tarhan G. Comparison and evaluation of LowensteinJensen medium and 2\% Ogawa medium for the diagnosis of tuberculosis. Mikrobiyol Bul. 2012;46(1):33-8.

20. Lubasi D, Habeenzu C, Mitarai S. Evaluation of an Ogawa mycobacterium culture method modified for higher sensitivity employing concentrated samples. Trop Med Health. 2004;32(1):1-4.

21. Hulley SB, Cummins SR, Browner WS, Grady D, Hearst N, Newman TB. Designing clinical research: an epidemiological approach. 2nd ed. Philadelphia: Lippincott Williams \& Wilkins; 2001.

22. Jullien S, Jain S, Ryan H, Ahuja V. Six-month therapy for abdominal tuberculosis. Cochrane Database Syst Rev. 2016;11:Cd012163.

\section{Publisher's Note}

Springer Nature remains neutral with regard to jurisdictional claims in published maps and institutional affiliations.

Ready to submit your research? Choose BMC and benefit from

- fast, convenient online submission

- thorough peer review by experienced researchers in your field

- rapid publication on acceptance

- support for research data, including large and complex data types

- gold Open Access which fosters wider collaboration and increased citations

- maximum visibility for your research: over $100 \mathrm{M}$ website views per year

At BMC, research is always in progress.

Learn more biomedcentral.com/submissions 